\title{
Functionalized
}

\section{nanoporous particles allow single-step purification of DNA reactions}

\section{The pitch}

Using a new materials-based approach, Diffinity Genomics is introducing a series of products for rapid, single-step purification of nucleic acid reactions such as polymerase chain reaction (PCR) amplification of DNA. The initial product, the Diffinity RapidTip ${ }^{\mathrm{TM}}$ for PCR Purification, removes unwanted materials from the PCR solution such as unincorporated oligonucleotides used as primers plus nucleotides (dNTP) thereby rendering the solution suitable for DNA sequencing reactions. Currently, PCR purification is done either by enzymes that selectively degrade the unwanted components (enzymatic cleanup) or by using differential solubility to selectively elute the desired double strands after immobilizing all of the nucleic acids (bind-wash-elute cleanup). Each of these processes takes 10-30 minutes and involves the addition of reagents. The approach taken by Diffinity Genomics is based on a single step in which the post-PCR solution is aspirated into a pipette tip containing functional material and the purified product dispensed. It is quicker than existing processes (60 seconds versus 10-30 minutes) and less
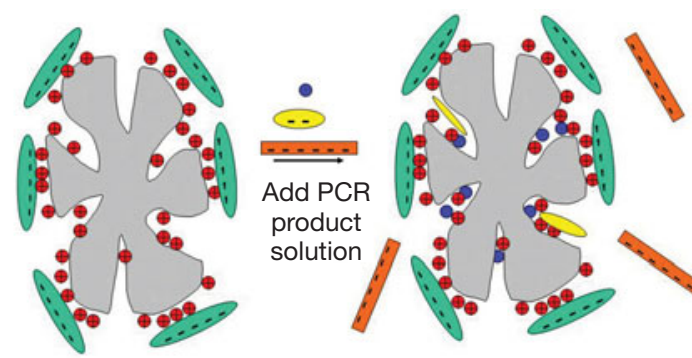

- Surface coating that captures dNTP and ss-DNA

Surface coating that blocks amplicon adsorption

- Unincorporated dNTP

$\ldots$ Unamplified primers

...... PCR Amplicons

Schematic depiction of differentially functionalized nanoporous particles for purification of polymerase chain reaction products. labor intensive. In addition, the need for reagents, additional consumables, or other infrastructure such as centrifuges, vacuum manifolds, or magnetic handlers is eliminated.

The current market for PCR cleanup products is over $\$ 200$ million per year. The customers are the approximately 400,000 researchers and laboratory technicians worldwide who study genomics or use it in their work. Diffinity's initial product, RapidTip ${ }^{\mathrm{TM}}$, improves workflow, is easier to use, and reduces operator-related errors thereby saving substantial time and labor costs. There is estimated to be a sizable number of researchers who currently use "home brew" PCR purification methods because of the expense and inconvenience of cleanup products.

\section{The technology}

The materials strategy enabling singlestep rapid purification is shown schematically in the figure where nanoporous particles can be functionalized so that small and large molecules can experience different surface adsorption characteristics. For this specific application, the pore interiors are selectively functionalized so that only the primers and unincorporated nucleotides in PCR product solutions can penetrate to be highly adsorbing while separately treating the particle exteriors experienced by the DNA amplicons (pieces of DNA formed by natural or synthetic amplification) to have distinct properties. This combination of size-exclusion chromatography with differential functionaliza- tion allows the rapid selective adsorption of the impurities while retaining the desired DNA in solution when the PCR product is exposed to the differentially functionalized nanoporous particles (DFNP).

When the particles are retained within a pipette tip designed for rapid mixing, the PCR purification process can be engineered to occur in 60 seconds or less and to produce DNA suitable for sequencing. The RapidTip is easily integrated into manual and automated liquid handling workflows. In addition, the company's molecular separation technology is readily scalable to very small microfluidic systems or adaptable to processes that handle large sample volumes.

One of the main applications of PCR amplified DNA is targeted sequencing where a particular region of the genome is selected for sequencing. Diffinity has demonstrated the efficacy of the RapidTip protocol for PCR cleanup prior to targeted sequencing.

Differentially functionalized nanoporous particles are a promising approach to many molecular separation problems. It is anticipated that the combination of rapid, single-pass molecular separation capability with the simplicity of the ubiquitous method for solution transfers pipette technology will enable the resulting functional pipettes to be widely applied in biotechnology and diagnostics.

\section{Opportunities}

Diffinity Genomics believes that its molecular separation technology can be leveraged into a variety of additional applications where rapid, single-pass or flow-through molecular separations are performed, such as microarrays, blood or water purification, simple diagnostic systems or larger scale industrial processes. The company welcomes and invites ideas for licensing new products and also joint research and development agreements beyond the scope of its present nucleic acid extraction and purification applications.

Source: Jeffrey Helfer, CEO Diffinity Genomics, 150 Lucius Gordon Drive, West Henrietta, NY 14586 USA; tel. 585-214-0595; fax 585-272-8142; e-mail jhelfer@diffinitygenomics.com; and www.diffinitygenomics.com.
} 